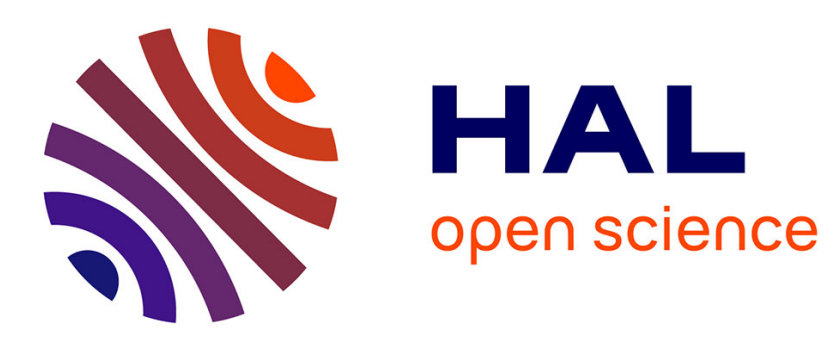

\title{
Short waves in ferromagnetic media
}

Hervé Leblond, Miguel Alberto Manna

\section{To cite this version:}

Hervé Leblond, Miguel Alberto Manna. Short waves in ferromagnetic media. Physical Review E : Statistical, Nonlinear, and Soft Matter Physics, 2009, 80 (3), pp.037602. 10.1103/PhysRevE.80.037602 . hal-03423713

\section{HAL Id: hal-03423713 \\ https://univ-angers.hal.science/hal-03423713}

Submitted on 10 Nov 2021

HAL is a multi-disciplinary open access archive for the deposit and dissemination of scientific research documents, whether they are published or not. The documents may come from teaching and research institutions in France or abroad, or from public or private research centers.
L'archive ouverte pluridisciplinaire HAL, est destinée au dépôt et à la diffusion de documents scientifiques de niveau recherche, publiés ou non, émanant des établissements d'enseignement et de recherche français ou étrangers, des laboratoires publics ou privés. 


\title{
Short waves in ferromagnetic media
}

\author{
H. Leblond ${ }^{1}$ and M. Manna ${ }^{2}$ \\ ${ }^{1}$ Laboratoire POMA, CNRS-FRE 2988, Université d'Angers, 2 Boulevard Lavoisier, 49045 Angers Cedex 1, France \\ ${ }^{2}$ Laboratoire de Physique Théorique et Astroparticules, IN2P3/CNRS-UMR 5207, Université Montpellier II, Place E. Bataillon, 34095 \\ Montpellier Cedex 05, France
}

(Received 19 December 2008; revised manuscript received 3 June 2009; published 18 September 2009)

\begin{abstract}
We discuss the propagation of nonlinear electromagnetic short waves in a magnetically saturated ferromagnetic thick film. The sample is magnetized to saturation by a field perpendicular to both the film plane and the propagation direction. A $(2+1)$ dimensional asymptotic model equation generalizing the sine-Gordon one is derived. Line soliton solutions are exhibited; their stability condition is derived. When unstable, line solitons decay into stable two-dimensional lumps, which are studied both numerically and analytically.
\end{abstract}

DOI: 10.1103/PhysRevE.80.037602

PACS number(s): 05.45.Yv, 41.20.Jb, 75.30.Ds

Electromagnetic wave propagation in a saturated ferromagnetic medium is usually described by the so-called Maxwell-Landau-Lifschitz model, which consists of the Maxwell equations, supplemented with the Landau-Lifschitz relation between the magnetization density $\mathbf{M}$ and the magnetic field $\mathbf{H}$. The resulting coupled system is highly nonlinear and dispersive, in contrast with nonmagnetic or paramagnetic materials, in which the usual constitutive relations are linear, and the wave equations can be solved exactly by Fourier methods. A lot of studies were devoted to approximate wave solutions of the Maxwell-Landau-Lifschitz system. They allow us to go round the fully nonlinear and dispersive system, not exactly solvable, and to derive intermediate asymptotic models [1] as it is proceeded in other physical contexts such as hydrodynamics, elasticity, and optics, i.e., using asymptotic expansions. Intermediate asymptotic models are valid at distances and times such that details and features of secondary importance to the phenomenon be negligible and vanish. At the same time they retain and account for the features essential for the comprehension of the phenomenon. This way nonlinear solitary wave propagation and Korteweg-de Vries (KdV)-type solitons have been theoretically described [2] and then extended to $(2+1)$ dimensions as solutions to the Kadomtsev-Petviashvili (KP) model [3,4].

Other kinds of intermediate asymptotic model can be obtained using the slowly varying envelope approximation (SVEA). The SVEA produces as an intermediate universal asymptotic model the nonlinear Schrödinger equation (NLS) [5]. Envelope solitons of NLS have been theoretically described and experimentally observed [6-10]. Dark solitons have also been predicted [10,11] and observed [12]. In (2 $+1)$ dimensions, NLS generalizes to the Davey-Stewartson system $[13,14]$.

All these studies were based on long-wave-type approximations: solitons of $\mathrm{KdV}$ or line solitons of KP have wavelengths larger than some typical scale of the sample and NLS represents dynamics of envelope waves of wavelengths larger than the underlying carrier's one. Consequently, these models and their solutions are useful to predict the behavior of large-scale phenomena.

Nowadays studies on short-wave-type approximations, started in the rather different context of hydrodynamics [15], have been adapted to be used in ferromagnetic media [16-18]. The continuation of this program is the objective of the present work. In Refs. $[16,17]$ the sine qua non condition that short waves propagate only in the direction perpendicular to the initial magnetization density $\mathbf{M}_{0}$ was found. We consider a ferromagnetic thick film lying in the $x y$ plane, a short wave propagating along $x$. In $[17,18], \mathbf{M}_{0}$ belonged to the plane of the film, as $\mathbf{M}_{0}=(0, m, 0)$, where $m$ is the normalized saturation magnetization. Such a configuration fulfils the short-wave propagation condition. Here we assume that the external magnetic field is perpendicular to the film, i.e., $\mathbf{M}_{0}=(0,0, m)$, which satisfies the propagation condition (Fig. 1). The questions we would like to answer in this work are: (a) how does the associated linear analysis change, (b) what is the $(2+1)$ asymptotic model equation (if any) induced by the new steady state, (c) how possible solutions behave faced with perturbations, and (d) does the background instability, discovered in [17], appear here ?

The thick film is magnetized to saturation by an external field $\mathbf{H}_{e x t}$ directed along $z$. The evolution of the magnetic field $\mathbf{H}$ is governed by the Maxwell equations, which reduce to

$$
-\boldsymbol{\nabla}(\boldsymbol{\nabla} \cdot \mathbf{H})+\Delta \mathbf{H}=c^{-2} \partial_{t}^{2}(\mathbf{H}+\mathbf{M}),
$$

where $c=1 / \sqrt{\mu_{0} \widetilde{\varepsilon}}$ is the speed of light with $\widetilde{\varepsilon}$ the scalar permittivity of the medium. The magnetization density $\mathbf{M}$ obeys the Landau-Lifschitz equation, which reads as

$$
\partial_{t} \mathbf{M}=-\gamma \mu_{0} \mathbf{M} \wedge \mathbf{H}+\frac{\sigma}{M_{s}} \mathbf{M} \wedge(\mathbf{M} \wedge \mathbf{H}),
$$

where $\gamma$ is the gyromagnetic ratio, $\mu_{0}$ is the magnetic permeability in vacuum, $\sigma<0$ is the damping constant, and $M_{s}$

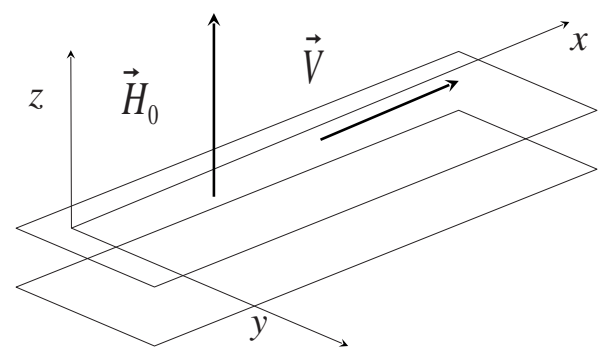

FIG. 1. The configuration considered. 
is the saturation magnetization. The internal magnetic field $\mathbf{H}$ is related to $\mathbf{H}_{\text {ext }}$ through

$$
\mathbf{H}=\mathbf{H}_{\text {ext }}-N \cdot \mathbf{M},
$$

where $N$ is the demagnetizing factor tensor. Here $N$ is diagonal with $\left(N_{x}, N_{y}, N_{z}\right)=(0,0,1)$.

We neglect inhomogeneous exchange since we consider bulk polaritons in a ferromagnet: in this case the wavelengths are large with regard to the exchange length. Surface anisotropy has a determinant effect on surface modes and in thin films. However, since the validity of the present study is restricted to volume modes in thick films, surface anisotropy can also be neglected. We also assume that the crystalline anisotropy of the sample is negligible. It can be conjectured that, for an easy axis perpendicular to the plane of the film, the crystalline anisotropy would not modify qualitatively the results of the present Brief Report. The quantities $\mathbf{M}, \mathbf{H}$, and $t$ are rescaled into $\mu_{0} \gamma \mathbf{M} / c, \mu_{0} \gamma \mathbf{H} / c$, and $c t$ so that the constants $\mu_{0} \gamma / c$ and $c$ in Eqs. (1) and (2) are replaced by 1, $M_{s}$ by $m=\mu_{0} \gamma M_{s} / c$, and $\sigma$ by $\widetilde{\sigma}=\sigma / \mu_{0} \gamma$, which is dimensionless.

In order to study the linear regime, we linearize Eqs. (1) and (2) about the steady state

$$
\mathbf{M}_{0}=(0,0, m), \quad \mathbf{H}_{0}=\left(0,0, H_{0}\right),
$$

where $m$ is the normalized saturation magnetization and $H_{0}$ $=(\alpha-1) m$ so that $\alpha$ is the strength of the external field in units of $M_{s}$. We seek for plane-wave solutions of the form

$$
\mathbf{M}=\mathbf{M}_{0}+\mathbf{m} e^{i \theta}, \quad \mathbf{H}=\mathbf{H}_{0}+\mathbf{h} e^{i \theta},
$$

where $\mathbf{m}$ and $\mathbf{h}$ are small constant vectors to be determined, and the phase is $\theta=k x+l y-\omega t$ where $k$ and $l$ are the wave numbers in the $x$ and $y$ directions and $\omega$ is the frequency.

Neglecting damping and linearizing Eqs. (1)-(5) about $\mathbf{M}_{0}, \mathbf{H}_{0}$, we obtain the dispersion relation

$$
\omega^{2}\left(\omega^{2}-k^{2}-l^{2}\right)-\alpha m^{2}\left(\omega^{2}+(\alpha-1)\left(\omega^{2}-k^{2}-l^{2}\right)\right)=0 .
$$

According to the short-wave approximation procedure [15], we introduce a small parameter $\varepsilon$ linked to the magnitude of the wavelength through $k=k_{0} / \varepsilon$. The direction of the wave propagation is assumed to be close to the $x$ axis in such a way that the $y$ variable gives only account of a slow transverse deviation. Therefore $l$ is assumed to be very small with respect to $k$ and we write $l=l_{0}$ of order 0 with respect to $\varepsilon$. We obtain

$$
\omega=\frac{k_{0}}{\varepsilon}+\frac{\alpha m^{2}+l_{0}^{2}}{2 k_{0}} \varepsilon+O\left(\varepsilon^{3}\right),
$$

which proves that the short-wave approximation is possible and motivates the introduction of the scaled variables

$$
\zeta=(x-V t) / \varepsilon, \quad y=y, \quad \tau=\varepsilon t .
$$

The variable $\zeta$ describes the shape of the wave propagating at speed $V$; it assumes a short wavelength about $1 / \varepsilon$. The slow time variable $\tau$ accounts for the propagation during very long time on very large distances with regard to the wavelength. The transverse variable $y$ has an intermediate scale, as in KP-type expansions [3,5]. In order to derive the nonlinear model, fields $\mathbf{M}$ and $\mathbf{H}$ are expanded in power series of $\varepsilon$ as

$$
\mathbf{M}=\mathbf{M}_{0}+\varepsilon \mathbf{M}_{1}+\varepsilon^{2} \mathbf{M}_{2}+\cdots,
$$

where $\mathbf{M}_{0}, \mathbf{M}_{1}, \ldots$,are functions of $(\zeta, y, \tau)$ and analogously for $\mathbf{H}$. They vanish at infinity except that $\mathbf{M}_{0}$ and $\mathbf{H}_{0}$ tend to $(0,0, m)$ and $(0,0,(\alpha-1) m)$, respectively. Expansion (9) and scaling (8) are substituted into Eqs. (1) and (2) and solved order by order. At leading order $\left[1 / \varepsilon^{2}\right.$ in Eq. (1) and $1 / \varepsilon$ in Eq. (2)] it is found that $\mathbf{M}_{0}$ is uniform, $H_{0}^{x}$ is zero, while $H_{0}^{y}$ and $H_{0}^{z}$ remain free if $V=1$. We consider now this value of the velocity $V$. At following order, we find that

$$
\mathbf{M}_{1}=-m \int_{-\infty}^{\zeta} H_{0}^{y} d \zeta^{\prime} \mathbf{e}_{x},
$$

with $\mathbf{e}_{x}$ being the unitary vector along $x$ and

$$
H_{1}^{x}=m \int_{-\infty}^{\zeta} H_{0}^{y} d \zeta^{\prime}-\int_{-\infty}^{\zeta} \partial_{y} H_{0}^{y} d \zeta^{\prime},
$$

while $H_{1}^{y}$ and $H_{1}^{z}$ are free.

At second order, after elimination of $M_{2}^{y}$ and $M_{2}^{z}$, we obtain

$$
\begin{gathered}
\partial_{\zeta} \partial_{y} H_{1}^{x}+\partial_{\zeta}\left(m H_{1}^{x}-M_{1}^{x} H_{0}^{z}\right)-2 \partial_{\zeta} \partial_{\tau} H_{0}^{y}=0, \\
\partial_{y}^{2} H_{0}^{z}-\partial_{\zeta}\left[M_{1}^{x} H_{0}^{y}\right]+2 \partial_{\zeta} \partial_{\tau} H_{0}^{z}=0 .
\end{gathered}
$$

Finally, eliminating $M_{1}^{x}$ and $H_{1}^{x}$ from Eqs. (12) and (13) with the help of Eqs. (10) and (11), we obtain evolution equations for $H_{0}^{y}$ and $H_{0}^{z}$ as

$$
\begin{gathered}
2 \partial_{\zeta} \partial_{\tau} H_{0}^{y}=\left(m^{2}-\partial_{y}^{2}\right) H_{0}^{y}+m \partial_{\zeta}\left(H_{0}^{z} \int_{-\infty}^{\zeta} H_{0}^{y} d \zeta^{\prime}\right), \\
2 \partial_{\zeta} \partial_{\tau} H_{0}^{z}=-\partial_{y}^{2} H_{0}^{z}-m \partial_{\zeta}\left(H_{0}^{y} \int_{-\infty}^{\zeta} H_{0}^{y} d \zeta^{\prime}\right) .
\end{gathered}
$$

Introducing dimensionless field and variables, system (14)(15) reduces to

$$
\begin{gathered}
C_{X T}=-B B_{X}+C_{Y Y}, \\
B_{X T}=B C_{X}+B_{Y Y} .
\end{gathered}
$$

with $X=-m x / 2, Y=m y, T=m t, H_{0}^{y}=-m B_{X}, H_{0}^{z}=-m\left(1+C_{X}\right)$, and the subscript denotes partial derivative.

System (16)-(17) coincides with the one derived in Refs. $[17,18]$ except that the transverse drift (terms involving $B_{Y}$ and $C_{Y}$ ) is absent. The scaling of the field and variables is the same except that $y$ and $z$ components are inverted, which is a natural consequence of the change in the direction of the applied field. Due to the applied field, the solutions of system (16)-(17) must satisfy the boundary conditions

$$
\lim _{X \rightarrow \infty} B_{X}=0, \quad \lim _{X \rightarrow \infty} C_{X}=-\alpha .
$$

Notice that the strength of the external field does not appear in the equations except through boundary condition (18). 

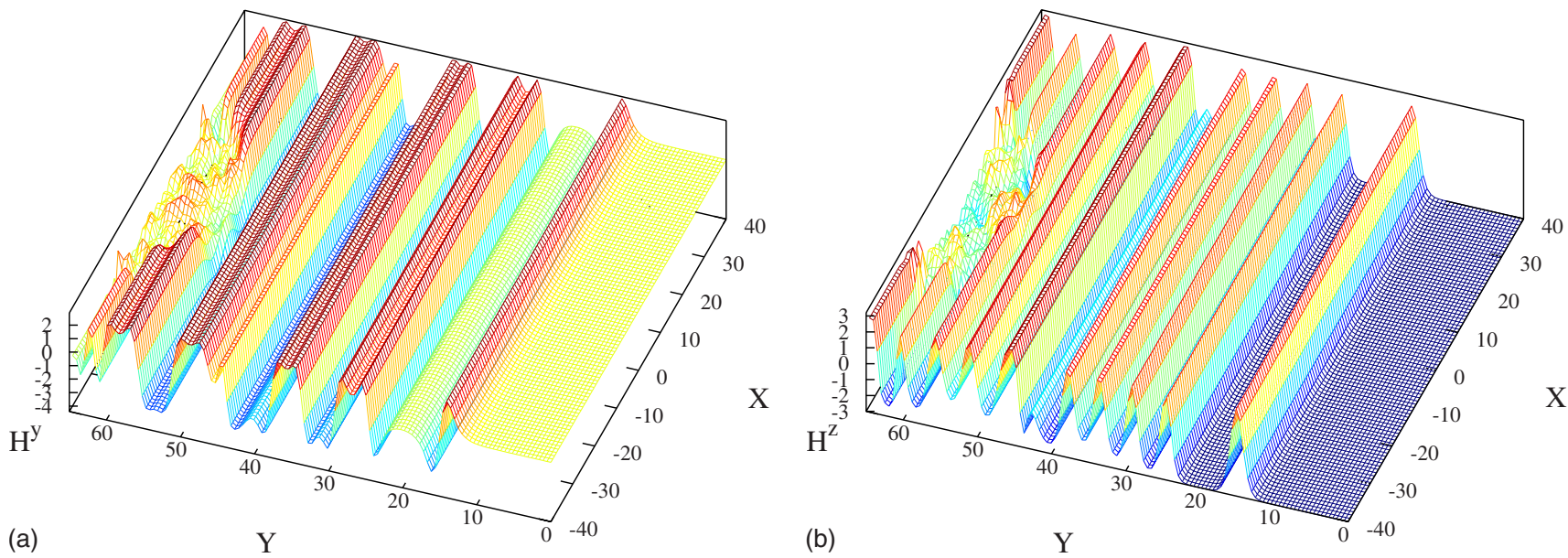

FIG. 2. (Color online) Propagation of an initially perturbed stable line soliton. (a) $H^{y}$ component; (b) $H^{z}$ component.

If transverse variations are neglected, system (16)-(17) reduces to the sine-Gordon (sG) equation as the system derived in the case of the in-plane field does. Hence from the kink solution of sG, the line soliton [17] is deduced,

$$
B=2 w \operatorname{sech} z, \quad C=w(2 \tanh z-z),
$$

where $z=X-w T$, with $w$ the soliton velocity.

The stability of line soliton (19) with respect to slow transverse perturbations is studied following the same approach as in [17]. We introduce a slow transverse perturbation $\theta$ of the variable $z$ and expand the fields in a perturbation series about the line soliton as

$$
B=B_{0}+\eta B_{1}+\cdots, \quad C=C_{0}+\eta C_{1}+\cdots,
$$

where $\eta$ is a small parameter and $B_{0}$ and $C_{0}$ have the expression given by Eq. (19), with $z$ perturbed as

$$
z=X-w T+\theta(\eta Y, \eta T) .
$$

The variables $\eta y$ and $\eta t$ are denoted by $\mathcal{Y}$ and $\mathcal{T}$, respectively. The expansion is then reported into system (16)-(17) and solved order by order. The expressions are the same as in [17] except that the terms proportional to $\theta_{y}$ in the equations and in the expressions of $B_{1}$ are absent. The final expression of $C_{1 z}$ is exactly the same. In each of the two equations obtained at order $\eta^{2}$, a term proportional to $\int z B_{1 y}$ is missing and so is the term proportional to $C_{1 \mathcal{Y}}$ is the second equation. Finally an equation of the same form is obtained,

$$
H \theta_{\mathcal{T} T}+I \theta_{\mathcal{Y Y}}+J \theta_{\mathcal{Y Y}}=0
$$

with the same $H=-2$, but $J=0$ and $I=-4 w$, instead of $J$ $=2 \pi$ and $I=-4(w+1)$ in [17]. Thus

$$
-2 \theta_{T T}-4 w \theta_{\mathcal{Y Y}}=0 .
$$

Then considering solutions of Eq. (23) of the form $\theta$ $=\exp (i \omega \mathcal{Y}+\lambda \mathcal{T})$, it is seen that $\lambda$ is purely imaginary and no instability occurs if $w<0$. As a conclusion, the line soliton is stable if its velocity $w$ is negative and unstable for $w>0$.

Comparing Eq. (18) with Eq. (19) shows that the speed $w$ of the line soliton must be equal to the strength $a$ of the external field. The stability condition $w<0$ for the line soliton corresponds to the assumption that the external field has the direction opposite to that of the saturated magnetization, which is known to be an unstable state.

In the case of the in-plane applied field, a transverse background instability occurs and can be removed by means of a narrowing of the sample [18]. No transverse background instability occurs here.

System (16)-(17) is solved numerically using the scheme presented in Ref. [17]. The propagation of an initially perturbed stable line soliton is shown on Fig. 2. It propagates backward in the moving frame. Numerical computation shows the arising of many other waves coming from infinity. It has been checked by using several modifications of the numerical method that this phenomenon is not a numerical artifact. It is related to the instability of the background, which is purely longitudinal here, in contrast with the case of the in-plane magnetization.

On the other hand, an initial unstable line soliton splits into stable two-dimensional lumps (see Fig. 3). They can be investigated by means of a variational approach. System (16)-(17) derives from the Lagrangian density

$$
\mathcal{L}=\frac{1}{2}\left(C_{X} C_{T}+B_{X} B_{T}-\left(C_{Y}\right)^{2}-\left(B_{Y}\right)^{2}+C_{X} B^{2}\right)
$$

through $\delta \mathcal{L} / \delta C=0$ and $\delta \mathcal{L} / \delta B=0$. We seek for traveling solutions of Eqs. (16) and (17), including a background field

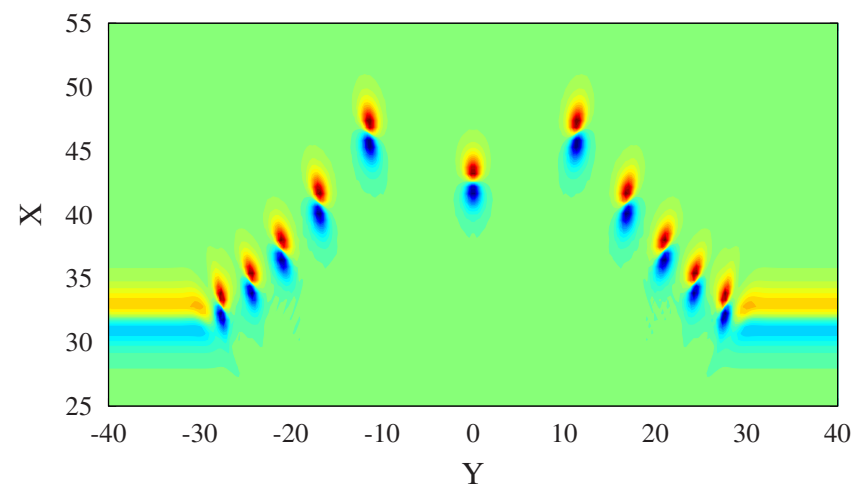

FIG. 3. (Color online) An initial unstable line soliton splits into lumps. 
$\mathbf{H}_{0}=[0,0,(\alpha-1) m] ;$ i.e., $\quad B=B(X-v T, Y)$ and $C=-\alpha X$ $+C^{\prime}(X-v T, Y)$. The equations are the same as in [17] except that the transverse drift terms $B_{Y}$ and $C_{Y}$ are absent. We compute an effective Lagrangian density and make use of the ansatz

$$
\begin{gathered}
B=p \exp \left(-X^{2} / f^{2}-Y^{2} / g^{2}\right), \\
C=-\mu X \exp \left(-X^{2} / f^{2}-Y^{2} / g^{2}\right) .
\end{gathered}
$$

The Lagrangian $L=\int_{\mathbb{R}^{2}} \mathcal{L}_{e f f} d X d Y$ is computed by standard methods and then a set of four equations is obtained by deriving $L$ with respect to the dynamical variables $p, \mu, f^{2}$, and $g^{2}$. It can be solved to yield

$$
\begin{gathered}
p^{2}=-9 \mu(1+3 q v) / 8 q, \quad \mu=-9 \alpha / 2, \\
f^{2}=(1+q v) / \alpha q, \quad g^{2}=q f^{2},
\end{gathered}
$$

with $q=(2+\sqrt{13}) / 3 v$. We have thus obtained a two parameter family of lumps, with the parameters being the applied field strength $\alpha$ and the lump velocity $v$; both $\alpha$ and $v$ must be positive. The variational lump is shown in Fig. 4. Numerical resolution of system (16)-(17), using the above variational approximation as an input, confirms the validity of the latter and proves the stability of the lumps, at least for the considered numerical examples.
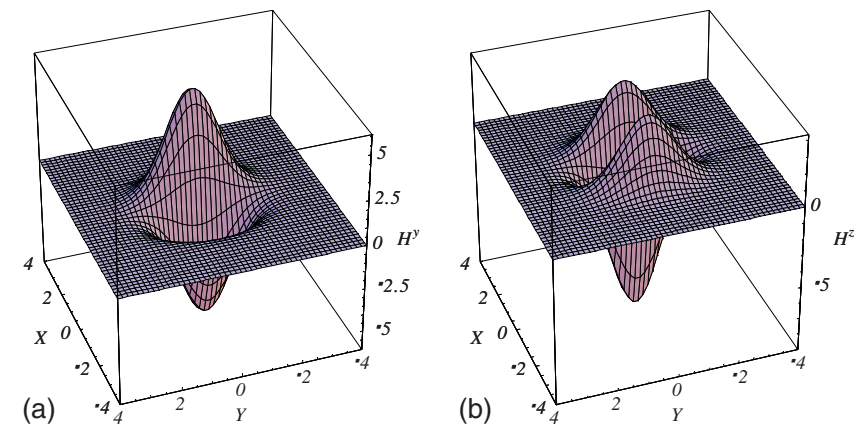

FIG. 4. (Color online) The variational lump. (a) $H_{y}$ component; (b) $H_{z}$ component $(\alpha=2, v=1$. Dimensionless).

As a conclusion, the main difference with the configuration with in-plane external field is the absence of the transverse drift. This feature simplifies the stability condition of the line solitons and suppresses the transverse instability of the background when they are stable. It also allows a more simple, explicit, and accurate description of the lumps by the variational analysis in the case of stable line solitons

This work is made in the framework of CNRS GDRPhoNoMi2 (Photonique Nonlinéaire et Milieux Microstructurés).
[1] G. I. Barenblatt, Scaling, Cambrigde Texts in Applied Mathematics (Cambridge University Press, Cambrigde, UK, 2003).

[2] H. Leblond, J. Phys. A 28, 3763 (1995); 36, 1855 (2003).

[3] B. B. Kadomtsev and V. I. Petviashvili, Sov. Phys. Dokl. 15, 539 (1970).

[4] H. Leblond, J. Phys. A 35, 10149 (2002).

[5] H. Leblond, J. Phys. B 41, 043001 (2008).

[6] A. K. Zvezdin and A. F. Popkov, Sov. Phys. JETP 57, 350 (1983).

[7] P. De Gasperis, R. Marcelli, and G. Miccoli, Phys. Rev. Lett. 59, 481 (1987)

[8] F. G. Bass, N. N. Nasonov, and O. V. Naumenko, Sov. Phys. Tech. Phys. 33, 742 (1988).

[9] B. A. Kalinikos, N. G. Kovshikov, and A. N. Slavin, Phys. Rev. B 42, 8658 (1990); A. N. Slavin and I. V. Rojdestvenski, IEEE Trans. Magn. 30, 37 (1994).

[10] H. Leblond and M. Manna, Phys. Rev. E 50, 2275 (1994).
[11] A. N. Slavin, Y. S. Kivshar, E. A. Ostrovskaya, and H. Benner, Phys. Rev. Lett. 82, 2583 (1999).

[12] Ming Chen, M. A. Tsankov, J. M. Nash, and C. E. Patton, Phys. Rev. Lett. 70, 1707 (1993).

[13] A. Davey and K. Stewartson, Proc. R. Soc. London, Ser. A 338, 101 (1974).

[14] H. Leblond, J. Phys. A 32, 7907 (1999).

[15] M. A. Manna and V. Merle, Phys. Rev. E 57, 6206 (1998); M. A. Manna, J. Phys. A 34, 4475 (2001).

[16] R. A. Kraenkel, M. A. Manna, and V. Merle, Phys. Rev. E 61, 976 (2000).

[17] M. Manna and H. Leblond, J. Phys. A 39, 10437 (2006); H. Leblond and M. Manna, Phys. Rev. Lett. 99, 064102 (2007); Phys. Rev. B 77, 224416 (2008).

[18] H. Leblond and M. Manna, J. Phys. A: Math. Theor. 41, 185201 (2008). 